\title{
Hysteroscopic management of intra-uterine devices with lost strings
}

Shubha Sagar Trivedi, MS, Professor; Madhu Goel, MD, Senior Resident; Sandhi Jain, MS, Senior Resident, Department of Obstetrics and Gynaecology, Lady Hardinge Medical College and Associated Hospitals, New Delhi, India

Correspondence: Dr SS Trivedi, Bungalow No. 22, Lady Hardinge Medical College, New Delhi-110001, India. Tel: 3360580, Email:drtrivedi@mantraonline.com

(Accepted October 26 $\left.{ }^{\text {th }}, 1999\right)$

\begin{abstract}
Summary
We report a series of 38 patients with intra-uterine devices with lost strings where hysteroscopic aid was required after routine retrieval procedures failed. Thirty-five intra-uterine devices could be removed easily with hysteroscope. In one patient a fragmented Lippes Loop was removed piecemeal hysteroscopically. Laparotomy was required in only one patient, for an extra-uterine Copper T. Hysteroscopy is thus a simple, safe and effective method for removing misplaced intra-uterine devices.
\end{abstract}

Key words

hysteroscopy, intra-uterine devices, lost strings

\section{Key message point \\ - Hysteroscopy is a safe and effective method for locating intra-uterine devices with lost strings.}

\section{Introduction}

With increased use of intra-uterine devices (IUDs) for contraception has come an increase in the number of related problems. ${ }^{1}$ A frequent clinical problem is the loss of the filament at the external cervical os, the 'lost tail'. The disappearance of the string or marker heralds potential problems such as retracted or torn off tail, misplacement within the cavity, intra-mural penetration or extra-uterine location. IUDs may be misplaced in as many as $5 \%$ of cases. $^{2}$

Procedures for retrieval of a misplaced device include extraction with a metal hook, artery forceps, cylindrical brush, ${ }^{3}$ thread retriever ${ }^{2}$ or dilatation and curettage. Success is not ensured with above methods; failure and uterine trauma may occur. Hysteroscopy as a diagnostic and operative technique has enabled safe retrieval of misplaced IUDs.

This communication reports our experience with hysteroscopy for managing the so called 'lost IUDs'.

\section{Method}

We present 38 cases with 'lost IUDs', referred to us after earlier repeated failed attempts at their removal. The age of the patients varied from 20-25 years with duration of use varying from $2.5-15$ years. In all 38 cases the presence of an IUD was confirmed either by ultrasound or X-ray. A $7 \mathrm{~mm}$ operative hysteroscope with grasping forceps was used for extraction of the IUD. Paracervical block was used in all cases with additional intravenous sedation in four cases.

\section{Results}

The IUDs retrieved were Copper Ts in 32 cases, Lippes Loops in three cases, and a stainless steel ring in one case. The position and characteristics of the IUDs on hysteroscopy are shown in Table 1.

In all 32 cases involving a Copper $\mathrm{T}$ the IUD was removed easily by grasping the vertical limb and
Table 1 Position and characteristics of IUDs on hysteroscopy

\begin{tabular}{lc}
\hline Hysteroscopic findings & No. of Patients \\
\hline $\mathrm{Cu}$ T in normal position & 26 \\
$\mathrm{Cu}$ T lying transversely & 2 \\
Transverse limb of Cu T in anteroposterior diameter & 2 \\
Fibroid below Cu T & 2 \\
Lippes Loop in normal position & 3 \\
Ring IUD & 1 \\
IUD not visualised & 2 \\
\hline
\end{tabular}

withdrawing the hysteroscope. Lippes Loop was visualised in three cases, two of which were removed easily, whilst the third was fragmented and was removed piecemeal with the hysteroscope. In one patient with postmenopausal bleeding, although hysteroscopy revealed no IUD, an embedded Lippes Loop was found in the posterior uterine wall on hysterectomy. In another case laparotomy revealed a Copper $\mathrm{T}$ embedded in the omentum just behind the uterus. In this case no IUD was visible on hysteroscopy despite an $\mathrm{X}$-ray reporting an intra-uterine IUD (Table 2).

Table 2 Removal of IUDs

\begin{tabular}{lc}
\hline Removal & Number of patients \\
\hline Easy Removal & 31 \\
$\quad$ Cu T & 3 \\
$\quad$ Lippes Loop & 1 \\
$\quad$ Ring IUD & 1 \\
Fragmented Lippes loop & 1 \\
Hysterectomy (for postmenopausal bleeding) & 1 \\
Laparotomy (for extra-uterine Cu T) & \\
\hline
\end{tabular}

No immediate or late complication of hysteroscopy was encountered. Two women complained of pain and needed intravenous sedation in addition to the paracervical block. One postmenopausal woman and one apprehensive woman were given sedation before the start of the procedure. Mild pain was reported by six women. Four women described the procedure very uncomfortable, nine were mildly uncomfortable and 15 described the procedure as comfortable.

\section{Discussion}

It is generally agreed that, once pregnancy has been excluded, proper investigations should be done to locate IUDs where threads are no longer visible. Appropriate methods are: sounding the uterine cavity with a probe; plain skiagram of abdomen and pelvis, AP and lateral view, with a probe or dilator in the uterus and ultrasonography. Blind manipulations with artery forceps, hooks and clamps for retrieval may be potentially dangerous, producing cervical or uterine injuries. ${ }^{1}$ Valle and Freeman ${ }^{1}$ advocated 
hysteroscopy as a primary method for locating and removing IUDs with missing tails in order to avoid unnecessary X-ray exposure and injuries by blind exploration. Siegler et al, ${ }^{4}$ however, recommended preliminary investigations for confirming the presence of IUDs in the uterus before hysteroscopy.

Recently, retrieval of IUDs under direct fluoroscopic control has been advocated by Broome and Torrie. ${ }^{5}$

Minimum hospital stay, comfort and avoidance of unnecessary radiation exposure makes hysteroscopy the preferred method for removal of misplaced IUDs. In our study we found hysteroscopy to be particularly useful in removal of fragmented devices.

\section{Conclusion}

We conclude that hysteroscopy is a useful and effective method in the localisation and retrieval of IUDs with missing tails.

Statements on funding and competing interests

Funding. None.

Competing interests. None.

References

Valle RF, Sciarra JJ, Freeman WD. Hysteroscopic removal of intrauterine devices with missing filaments. Obstet Gynecol 1977; 49: 55.

(isplaced intrauterine device. Aust NZ J Obstet Gynaecol 1996; 36: 49-51.

Ben-Rafael Z, Bider D. A new procedure for removal of a 'lost' intrauterine device. Obste Gynecol 1996; 87: 785-786.

Siegler AM, Kemmann E. Location and removal of misplaced or embedded intrauterine device by hysteroscopy. J Reprod Med 1976; 16: 139-144. Broome M, Torrie P. A new way of retrieving lost IUDs under direct fluoroscopic control. $\mathrm{Br}$. 